\title{
Panax notoginseng saponins prevent senescence and inhibit apoptosis by regulating the PI3K-AKT-mTOR pathway in osteoarthritic chondrocytes
}

\author{
YUBIAO ZHANG, WEISONG CAI, GUANGTAO HAN, SIQI ZHOU, JIANPING LI, MAO CHEN and HAOHUAN LI
}

Department of Orthopedics, Renmin Hospital of Wuhan University, Wuhan, Hubei 430060, P.R. China

Received September 18, 2019; Accepted January 24, 2020

DOI: $10.3892 /$ ijmm.2020.4491

\begin{abstract}
Panax notoginseng saponins (PNS) are active extracts obtained from the $P$. notoginseng plant. PNS exhibit various anti-inflammatory, anti-oxidant and anti-aging pharmacological properties in some cells. However, the effects of PNS on senescence and apoptosis in chondrocytes have not been studied to date. In the present study, whether PNS could limit tumor necrosis factor (TNF)- $\alpha$-induced senescence and apoptosis in chondrocytes and whether they could slow down cartilage degeneration in a surgery-induced rat osteoarthritis (OA) model by regulating the phosphatidyl inositol 3 kinase (PI3K)-protein kinase B (AKT)-mammalian target of rapamycin (mTOR) signaling pathway was examined. A potential mechanism underlying these effects was further elucidated. The present in vitro experiments showed that PNS significantly inhibited senescence and apoptosis in OA chondrocytes and prevented a decrease in the mitochondrial membrane potential and excessive mitochondrial permeability. In addition, the expression levels of autophagy-related proteins and the anti-apoptotic protein Bcl-2 were significantly increased in PNS-treated OA chondrocytes, but the expression levels of Bax and caspase- 3 were decreased; these effects were concentration-dependent. TNF- $\alpha$ significantly increased the expression of $\mathrm{p}-\mathrm{PI} 3 \mathrm{~K} / \mathrm{p}-\mathrm{AKT} / \mathrm{p}-\mathrm{mTOR}$ in OA chondrocytes, whereas PNS reduced PI3K, AKT and mTOR phosphorylation. The results of the in vivo experiments demonstrated that PNS significantly inhibited the PI3K-AKT-mTOR signaling pathway and collagen II degradation, as well as reduced matrix metalloproteinase (MMP)-3 and MMP-13 expression in chondrocytes in a rat OA model, thus attenuating cartilage destruction in OA. The results obtained in the rat model were consistent with the in vitro experimental results.
\end{abstract}

Correspondence to: Professor Haohuan Li, Department of Orthopedics, Renmin Hospital of Wuhan University, 99 Zhangzhidong Road, Wuchang, Wuhan, Hubei 430060, P.R. China E-mail: lihaohuan@whu.edu.cn

Key words: osteoarthritis, chondrocyte, Panax notoginseng saponins, phosphatidyl inositol 3 kinase-protein kinase B-mammalian target of rapamycin autophagy pathway, senescence
Furthermore, histological analyses and ultrastructural observations confirmed these results. Taken together, the results of the present study demonstrated that PNS may protect osteoarthritic chondrocytes from senescence and apoptosis by inhibiting the PI3K-AKT pathway, thus delaying the degradation of articular cartilage.

\section{Introduction}

Senescence and trauma are major risk factors contributing to the development of osteoarthritis (OA), a serious and debilitating chronic disease (1). Arthritis is characterized by the degeneration of articular cartilage, destruction of the extracellular matrix (ECM) and even complete loss of function. Numerous studies have confirmed that increased age is associated with radiographic changes and high prevalence of OA-related symptoms in the hands, hip joints, spine and knee joints (2-4). Articular cartilage undergoes aging-related structural and biochemical changes, which impair its biomechanical functions and lead to structural abnormality in the cartilage (5). Senescence-dependent changes in the cartilage include decreased chondrocyte density and compromised proliferation/biosynthetic function (6). With increased age, more senescent cells accumulate in the human body and secrete inflammatory cytokines, chemokines and proteases, collectively known as senescence-related secretory phenotypes (7). Senescent cells secrete a senescence-associated secretory phenotype (SASP) to alter the tissue microenvironment, attract immune cells and induce malignant phenotypes in adjacent cells (8). Constant renewal of cellular components occurs in tissues with high cellular turnover rates (9). However, chondrocytes are the only type of cells in the adult articular cartilage in which regeneration and renewal abilities are highly limited (10); additionally, such capability declines with age. The rate of chondrocyte division after mitosis is hardly detectable (11). OA-related loss of cartilage homeostasis leads to continued ECM destruction (12). Therefore, studies investigating the mechanisms of chondrocyte senescence and apoptosis may provide a new perspective to preserve cartilage integrity and prevent OA (13).

Autophagy regulates physiological functions, such as remodeling, differentiation, survival, death and senescence (14). As a cytoprotective mechanism, autophagy protects organisms from senescence by regulating the turnover of proteins 
and clearance of dysfunctional organelles. For example, autophagy is activated to sustain highly energy-dependent processes and low-nutrient energy metabolism, e.g., growth and differentiation. The regulatory mechanisms of autophagy are diverse. The regulation of mammalian target of rapamycin (mTOR), including activation of PI3K and TOR complex 1 , takes a key role in activation of autophagy. The phosphatidyl inositol 3 kinase (PI3K)-protein kinase B (AKT) pathway transmits a signal to TORC1, leading to mTOR activation, which then regulates the activity of autophagy. Therefore, the PI3K-AKT pathway is considered to be one of the most important upstream pathways of mTOR (15). It is widely believed that the PI3K-AKT-mTOR pathway is an essential cellular signaling pathway that negatively regulates autophagy after its activation. Autophagy plays a fundamental regulatory role in the pathological progression of inflammatory diseases (16). It has been observed that the deficiencies in autophagy and regulation of articular cartilage and cartilage cells can lead to senescence of the joints and, finally in turn, OA. Consequently, autophagy is considered to be a key factor in cartilage degradation and is an important mechanism that leads to OA (17). Previous studies have demonstrated that the basal level of autophagy decreases with age $(18,19)$, suggesting that enhancing autophagy might be an effective way to delay the degeneration of articular cartilage. However, the exact mechanism underlying the link between autophagy and OA has not yet been clarified. Therefore, the purpose of the present study is to develop possible beneficial treatment strategies for $\mathrm{OA}$ via regulation of the PI3K/AKT pathway.

Panax notoginseng saponins (PNS) is a mixture that contains ginsenosides such as Rb1, Rc, Rd, Re, Rg1 and Rh1 (20). PNS exerts various pharmacological functions, including hemostasis maintenance and anti-leukemia effects, as well as anti-tumor, anti-atherosclerosis, anti-inflammatory, anti-oxidative and anti-apoptotic effects (20). The important role of PNS in preventing cell senescence has been confirmed (21). Numerous components (Re, Rb1 and Rg1) present in PNS can prevent and treat a variety of diseases, such as coronary artery disease, neurodegenerative disease, diabetes and acute pancreatitis, by regulating autophagy (20-24). PNS can inhibit pancreatitis by inhibiting the PI3K/AKT pathway, promoting mTOR expression and inhibiting autophagy in pancreatic cells (24). Since the PI3K/AKT/mTOR pathway is also important in the occurrence and development of OA, reduced expression of the PI3K/AKT/mTOR pathway components may increase autophagy in chondrocytes and prevent their entry into senescence, thus preventing OA. However, the roles of PNS in chondrocyte senescence and autophagy regulation have not been confirmed. The aim of the present study was to explore the mechanisms by which PNS may prevent chondrocyte senescence via increasing autophagy and reducing apoptosis by regulating the $\mathrm{PI} 3 \mathrm{~K} / \mathrm{AKT} / \mathrm{mTOR}$ pathway.

\section{Materials and methods}

Experimental animals. All animal experiments were carried out in accordance with the regulations of the Administration of Laboratory Animals promulgated by the National Science and Technology Commission of the People's Republic of China and the Institute of Medical Ethics of Wuhan University Medical College. Sprague-Dawley (SD) rats were obtained from the Animal Experimental Center of Wuhan University (Wuhan, China). The present study was approved by the Laboratory Animal Welfare \& Ethics Committee of the Renmin Hospital of Wuhan University (Wuhan, China). All efforts were made to minimize the suffering of the animals used for the present experiments.

Cell culture and identification. A total of five healthy male SD rats (8 weeks old, weighing 220-240 g) were used in the present study. The animals were kept in a stable environment (12-h light/dark cycle, $25 \pm 3^{\circ} \mathrm{C}, 35-60 \%$ relative humidity with rat feed and tap water available ad libitum) for 3 weeks prior to sacrifice. The rats were sacrificed and the articular cartilage was carefully harvested. The specimens were washed three times with $10 \mathrm{ml}$ PBS and then minced into $1 \mathrm{~mm}^{3}$ sections. The rat cartilage sections were digested with $3 \mathrm{ml}$ of $0.25 \%$ trypsin for $40 \mathrm{~min}$ and with $0.2 \%$ collagenase II for further $6 \mathrm{~h}$. Next, the cells were collected by centrifugation at $1,000 \mathrm{xg}$ for $5 \mathrm{~min}$ at room temperature, incubated at $37^{\circ} \mathrm{C}$ with $5 \% \mathrm{CO}_{2}$ for $5 \mathrm{~min}$, mixed with $5 \mathrm{ml}$ DMEM (Gibco; Thermo Fisher Scientific, Inc.) with 10\% FBS (Gibco; Thermo Fisher Scientific, Inc.) and $1 \%$ mixture of penicillin and streptomycin and cultured in an incubator at $37^{\circ} \mathrm{C}$ with $5 \% \mathrm{CO}_{2}$. Live chondrocytes were identified by toluidine blue staining at $37^{\circ} \mathrm{C}$ for $30 \mathrm{~min}$.

Cell viability at different concentrations of PNS treatment. Cells were cultured in triplicate in 96-well plates $(8,000$ cells/well). To quantify cell viability at a specified time, $10 \mu 1$ Cell Counting Kit-8 (CCK-8) reagent (Beyotime Institute of Biotechnology) was added to each well and incubated at $37^{\circ} \mathrm{C}$ for $2 \mathrm{~h}$ according to the manufacturer's protocol. Optical density (OD) was detected using an automatic microplate reader at $450 \mathrm{~nm}$. The OD450 value is directly proportional to cell viability. All experiments were performed in triplicate in three independent experiments.

Experimental grouping of rat chondrocytes. The rat chondrocytes were divided randomly into 4 groups $(\mathrm{n}=3)$ : Control, $\mathrm{OA}$, OA + PNS (100 $\mu \mathrm{g} / \mathrm{ml})$ [Kunming Pharmaceutical Company (Lot no. 12JB09); Patent no. ZL96101652.3] and OA + PNS $(200 \mu \mathrm{g} / \mathrm{ml})$. The chondrocytes were cultured in 6-well culture plates $\left(1 \times 10^{6}\right.$ cells/well) for $24 \mathrm{~h}$. Untreated chondrocytes were used as a control group. The OA model phenotype was induced by treating normal cells with tumor necrosis factor (TNF)- $\alpha(20 \mathrm{ng} / \mathrm{ml})$ (Peprotech, Inc.). After $36 \mathrm{~h}$ of intervention, different concentrations of PNS (low concentration, $100 \mu \mathrm{g} / \mathrm{ml}$; high concentration, $200 \mu \mathrm{g} / \mathrm{ml}$ ) were added to the OA chondrocytes, followed by further incubation for $24 \mathrm{~h}$.

$S A-\beta$-galactosidase staining. The chondrocytes $\left(1 \times 10^{6}\right.$ cells/well $)$ were washed twice and fixed by adding $1 \mathrm{ml}$ of $1 \mathrm{X}$ fixative, followed by incubation for $10 \mathrm{~min}$ at $25^{\circ} \mathrm{C}$. Subsequently, the fixed cells were washed three times with PBS, stained using a SA- $\beta$-galactosidase staining kit (Beyotime Institute of Biotechnology) according to the manufacturer's protocol and incubated overnight at $37^{\circ} \mathrm{C}$. Finally, the cells were observed under a fluorescence microscope (Olympus Corporation). 
Reverse transcription-quantitative $(R T-q) P C R$ analysis. Total RNA was extracted from chondrocytes with TRIzol reagent (Invitrogen; Thermo Fisher Scientific, Inc.). The RevertAid First Strand cDNA Synthesis kit (Fermentas; Thermo Fisher Scientific, Inc.) was used to reverse transcribe cDNA from the RNA at $37^{\circ} \mathrm{C}$ for $15 \mathrm{~min}$ and $85^{\circ} \mathrm{C}$ for $5 \mathrm{sec}$. Quantitative PCR was performed using a StepOnePlus device (Applied Biosystems; Thermo Fisher Scientific, Inc.) at $95^{\circ} \mathrm{C}$ for $10 \mathrm{sec}$, followed by 40 cycles of $95^{\circ} \mathrm{C}$ for $5 \mathrm{sec}$ and at $60^{\circ} \mathrm{C}$ for $20 \mathrm{sec}$. The following primers were used: COL2 forward, $5^{\prime}$-AGGGCC AGGATGTCCGGCA-3' and reverse, 5'-GGGTCCCAGGTT CTCCATCT-3'; MMP-3 forward, 5'-CGGTGGCTTCAGTAC CTTTC-3' and reverse, 5'-ACCTCCTCCCAGACCTTCA-3'; MMP-13 forward, 5'-GGAGCATGGCGACTTCTAC-3' and reverse, 5'-GAGTGCTCCAGGGTCCTT-3'; and GADPH forward, 5'-AGGTCGGTGTGAACGGATTTG-3' and reverse, 5'-GGGGTCGTTGATGGCAACA-3'. GADPH was used as an internal reference. The $2^{-\Delta \Delta \mathrm{Cq}}$ method was used to calculate the relative mRNA expression levels (25).

Flow cytometry to measure mitochondrial membrane potential (MiMP). The JC-1 assay kit (Beijing Solarbio Science \& Technology Co., Ltd.) was used to evaluate MiMP. The minced cartilage pieces were washed with PBS, digested with trypsin and were incubated with $800 \mathrm{ml}$ the solution for $20 \mathrm{~min}$ at $37^{\circ} \mathrm{C}$. MiMP was measured using a FACSCalibur flow cytometer (Becton-Dickinson and Company) after the cells were washed and suspended in $1 \mathrm{ml} 1 \mathrm{X} \mathrm{JC}-1$ staining buffer. FlowJo 10 software was used for analysis (Becton-Dickinson and Company).

Detection of mitochondrial membrane permeability conversion (MPTP) openness. A working solution was prepared according to protocol provided in the MPTP detection kit (Best-Bio Ltd.), and $5 \mu \mathrm{l}$ fluorescence quencher and $3 \mu \mathrm{l}$ working solution of the probe were added to each sample. Staining was carried out in the dark at $25^{\circ} \mathrm{C}$ for $20 \mathrm{~min}$. After washing with $1 \mathrm{ml}$ MPTP buffer, MPTP was measured by flow cytometry as described above. The MPTP openness was evaluated based on the number of fluorescent units.

Flow cytometry to assess apoptosis. The rate of apoptosis was assessed by flow cytometry. Briefly, cells were separated from the culture supernatant by centrifugation at $2,000 \mathrm{xg}$ for $5 \mathrm{~min}$ at room temperature and then washed twice with pre-chilled PBS. The chondrocytes were carefully suspended in $500 \mu \mathrm{l}$ of binding buffer. Next, $5 \mu \mathrm{l}$ propidium iodide (PI) and $5 \mu \mathrm{l}$ fluorescein isothiocyanate (FITC)-labeled Annexin V (Becton-Dickinson and Company) were added, followed by staining at room temperature for $15 \mathrm{~min}$ in the dark, and the apoptotic rate was estimated by flow cytometry (Becton-Dickinson and Company).

Microscopic observation of MiMP. MiMP was evaluated using a Mitochondrial Membrane Potential Assay kit (Beijing Solarbio Science \& Technology Co., Ltd.), according to the manufacturer's protocol. After washing, chondrocytes were stained with $800 \mu \mathrm{l}$ working solution JC-1 at $37^{\circ} \mathrm{C}$ for $25 \mathrm{~min}$. After removing the staining solution, the cells were added to $2 \mathrm{ml}$ DMEM with $10 \%$ FBS. The red-green fluorescence ratio was observed under an Olympus fluorescence microscope (Olympus Corporation).

Immunofluorescence and immunohistochemistry. After washing with PBS, the specimens were fixed in $4 \%$ paraformaldehyde and permeabilized for $10 \mathrm{~min}$ at room temperature. Next, primary antibodies against P62 (1:200; Abcam; cat. no. ab155686) and COL-II (1:200; Abcam; cat. no. ab34712) were added and incubated in the dark at $25^{\circ} \mathrm{C}$ for $1 \mathrm{~h}$. The samples were then incubated with FITC-conjugated anti-mouse (1:200; Abcam; cat. no. ab6785) and Cy3-conjugated goat anti-rabbit (1:300; cat. no. GB21303; Servicebio, Inc.) secondary antibody at $37^{\circ} \mathrm{C}$ for $1 \mathrm{~h}$ and subsequently incubated with DAPI (Nanjing KeyGen Biotech Co., Ltd.) at $37^{\circ} \mathrm{C}$ for $5 \mathrm{~min}$. The cells were observed under an Olympus fluorescence microscope and positive expression was indicated by green (p62) and red (COL-II) staining. Image Pro Plus 6.0 analysis software (Media Cybernetics, Inc.) was used to analyze the proportions of positive cells in the samples.

The tissue sections were incubated with primary antibodies against MMP-3 (1:200; Abcam; cat. no. ab52915) or MMP-13 (1:200; Abcam; cat. no. ab39012) overnight at $4^{\circ} \mathrm{C}$ and subsequently incubated with a biotinylated Goat anti-rabbit secondary antibody (1:200; Abcam; cat. no. ab205718). The cells were observed under an Olympus light microscope and positive expression was indicated by brown staining. Image Pro Plus 6.0 analysis software (Media Cybernetics, Inc.) was used to analyze the proportions of positive cells in the samples.

Animal model and PNS treatment. The rats were randomized into 4 groups $(n=5)$ : control, OA, OA+PI3K-AKT-mTOR pathway agonist IGF-1 (10 mg/kg), OA+IGF-1+PNS $(200 \mathrm{mg} / \mathrm{kg})$. The OA rat model was constructed using resection of the medial meniscus of the right knee and transection of the anterior cruciate ligament. The animals were placed in an electric coil cage for $1 \mathrm{~h}$ every $24 \mathrm{~h}$. One month later, IGF-1 $(10 \mathrm{mg} / \mathrm{kg})$ and $40 \mu \mathrm{l} \mathrm{PNS}(75 \mu \mathrm{mol} / \mathrm{l})$ were injected into the joints of rats as per the appropriate treatments of each group (IGF-1 and PNS were injected into rat joints every three days for a total of 8 weeks), while OA and control groups received saline injections. All of the experimental rats were sacrificed 3 months after surgery.

Ultrastructural chondrocyte detection. The cartilage sections were sectioned into $1 \mathrm{~mm}^{3}$ pieces, fixed with $2.5 \%$ glutaraldehyde for $2 \mathrm{~h}$ at $4^{\circ} \mathrm{C}$ and washed with PBS. A concentration gradient of $1 \%$ Ottoman acid in ethanol (50, 70, 90 and 100\%) was used to fix the samples. The samples were immersed in a mixture of acetone and epoxy resin twice at $37^{\circ} \mathrm{C}(2: 1$ for $3 \mathrm{~h}$ the first time, 1:2 overnight the second time). Finally, the tissues were embedded in epoxy resin-filled capsules and heated at $70^{\circ} \mathrm{C}$ overnight. Ultrathin sections $(60-80 \mathrm{~nm})$ were sliced and observed using a transmission electron microscope (Hitachi, Ltd.) at x100,000 magnification.

Western blot analysis. Phosphatase inhibitor: Protease inhibitor: RIPA lysate was mixed at a ratio of (1:1:50) and added to cells or cartilage fragments to extract total proteins (Google Biotechnology). The proteins (40 $\mu \mathrm{g} /$ lane) were separated by SDS-PAGE on a $12 \%$ gel and then transferred 
to PVDF membranes. The membranes were blocked with $5 \%$ nonfat milk in TBS with $0.05 \%$ Tween-20 (TBST for $1 \mathrm{~h}$ at $37^{\circ} \mathrm{C}$ and incubated overnight at $4^{\circ} \mathrm{C}$ with the following primary antibodies: Interleukin (IL)-1 $\beta(1: 1,000$; cat. no. 12703; Cell Signaling Technology, Inc.), high mobility group protein B1 (HMGB1; 1:1,000; cat. no. 10829-1-AP; Proteintech, Inc.), caspase 8 (1:1,000; cat. no. 4790), p16 (1:1,000; cat. no. 80772), PI3K (1:1,000; cat. no. 4249), AKT (1:1,000; cat. no. 4691; all from Cell Signaling Technology, Inc.), phosphorylated (p-)AKT (1:500; cat. no. ab38449), p-PI3K (1:500; cat. no. ab182651; both from Abcam), mTOR (1:1,000; cat. no. 2983; Cell Signaling Technology, Inc.), p-mTOR (1:500; cat. no. ab84400; Abcam,), Bcl-2 (1:1,000; cat. no. 3498; Cell Signaling Technology, Inc.), Bax (1:1,000; cat. no. ab32503; Abcam), caspase-3 (1:1,000; cat. no. 9662; Cell Signaling Technology, Inc.), Beclin-1 (1:1,000; cat. no. 3495; Cell Signaling Technology, Inc.), LC3 (1:1,000; cat. no. 14600-1-AP; Proteintech, Inc.) and GAPDH (1:5,000; cat. no. GB12002; Servicebio, Inc.), Next, the membranes were washed three times in TBST and incubated with a horseradish peroxidase-conjugated goat anti-rabbit (1:3,000; cat. no. GB23303; Servicebio, Inc.) secondary antibody. After washing with TBST, a chemiluminescence luminol reagent (cat. no. G2014, Servicebio, Inc.) was used to visualize the protein bands employing the Image Lab 5.2 quantitative assay system (Bio-Rad Laboratories, Inc.). The relative protein levels were determined by normalizing to GAPDH.

Statistical analysis. Data are expressed as the mean \pm standard deviation for each group. Data analysis was conducted using SPSS 21.0 (IBM Corp.) and GraphPad Prism 6.0 (GraphPad Software, Inc.). Comparisons among multiple groups were performed using one-way analysis of variance with Dunnett's post hoc test. $\mathrm{P}<0.05$ was considered to indicate a statistically significant difference. GraphPad Prism software version 5.0 (GraphPad Software, Inc.) was used to plot the data.

\section{Results}

Culture and identification of chondrocytes and effect of PNS on TNF- $\alpha$-induced expression of senescent markers in cultured OA chondrocytes. Chondrocytes are either spindle-shaped, polygonal, or irregularly shaped with multiple protrusions. Positive toluidine blue staining can be used to identify the chondrocytes, as they are stained blue-purple. The cytoplasm of chondrocytes contained blue-purple heterochromatic granules, indicating that they expressed proteoglycan (Fig. 1A). The CCK-8 reagent was used to detect the cell viability of different concentrations of PNS, thereby determining the PNS concentration in the experiment was 100 and $200 \mu \mathrm{g} / \mathrm{ml}$. Cell viability at different concentrations of PNS. The viability of OA chondrocytes gradually increased when PNS concentration ranged between 0 and $200 \mu \mathrm{g} / \mathrm{ml}$; cell viability was inhibited when PNS concentration exceeded $200 \mu \mathrm{g} / \mathrm{ml}$ (Fig. 1B). Rat chondrocytes were divided into four groups: Control, OA, OA+PNS $(100 \mu \mathrm{g} / \mathrm{ml})$ and OA+PNS $(200 \mu \mathrm{g} / \mathrm{ml})$. Western blotting was used to detect and analyze the levels of SASP factors (IL-1 $\beta$, HMGB1, caspase- 8 and p16). The percentages of SASP cells and SA- $\beta$-gal-positive cells were highest in the
OA group compared with the other groups. However, PNS significantly reduced the expression levels of aging-related markers in OA chondrocytes in a concentration-dependent manner (Fig. 1C-F).

PNS can improve mitochondrial dysfunction and morphological changes and has an anti-apoptosis effect in $O A$ chondrocytes. Mitochondria play important roles in energy production, cell signaling, differentiation, apoptosis and senescence. Cell death and senescence often cause loss of MiMP and opening of MPTPs. Therefore, the effects of PNS on MiMP were examined by fluorescence microscopy (the red/green ratio indicates the expression of MiMP) (Fig. 2A). Flow cytometry was used to detect changes in MiMP (Fig. 2B and C), and MPTP (Fig. 2D). The results of these experiments showed that the OA group had the lowest MiMP, the highest MPTP openness and the highest apoptosis rate. PNS increased MiMP and decreased MPTP opening and apoptosis. These results indicate that PNS protects mitochondria of the chondrocytes.

PNS can significantly reduce the level of apoptosis in $O A$ chondrocytes. Chondrocyte apoptosis is an important process in the development of OA. In the present research, different methods were used to detect the degree of chondrocyte apoptosis. Flow cytometry was used to detect changes in the rate of apoptosis (Fig. 3A and B). Western blotting was used to detect and analyze the levels of the apoptosis-related factors caspase-3, Bax and Bcl-2 (Fig. 3C and D). The results of the flow cytometry experiments were consistent with those of western blot experiments. The expression of caspase- 3 and Bax was increased in the OA group compared with those in the control group; however, the level of the anti-apoptotic protein Bcl-2 was significantly decreased. These trends were reversed after the addition of PNS. PNS treatment significantly reduced caspase-3 and Bax levels but enhanced Bcl-2. These results indicate that PNS counteracts apoptosis.

$P N S$ reduces chondrocyte apoptosis by regulating the PI3K/AKT/mTOR pathway to increase the expression levels of autophagy-related proteins. The expression levels of PI3K, p-PI3K, AKT, mTOR, p-AKT, p-mTOR and the autophagy-related proteins LC3 and Beclin-1 were assessed by western blotting, and the expression of P62 in chondrocytes was measured by immunofluorescence staining. The phosphorylation levels of PI3K, AKT and mTOR were significantly increased in the other groups compared to those in the control group. In contrast, the expression levels of LC3II/LC3I, Beclin-1 and P62 were significantly decreased. Compared with those in the OA group, PNS significantly reduced the phosphorylation levels of p-PI3K, p-AKT and p-mTOR (Fig. 4A and B) and significantly increased the levels of autophagy-related proteins (Fig. 4C-G) in a concentration-dependent manner. Taken together with the previous experimental results, it can be concluded that PNS can reduce autophagy in chondrocytes and reduce apoptosis by regulating mTOR signaling via the PI3K-AKT pathway.

PNS regulates the expression levels of degeneration-related genes and ultrastructural changes in cartilage. The 
A

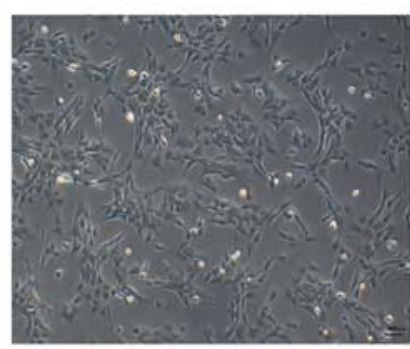

C

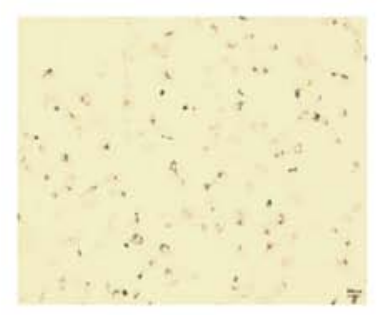

Control

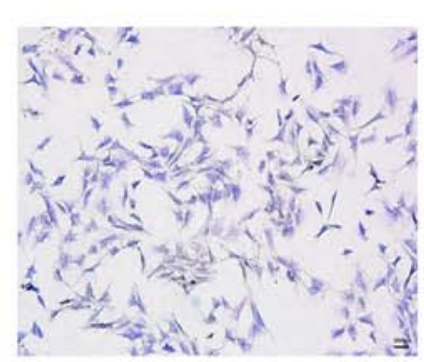

B

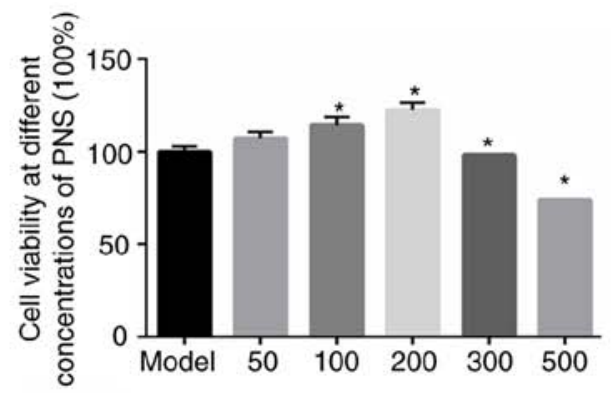

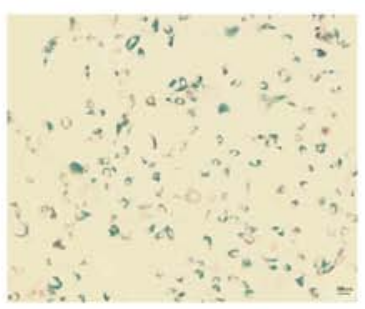

$\mathrm{OA}$

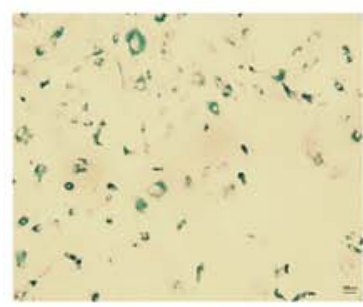

OA+PNS $(100 \mu \mathrm{g} / \mathrm{ml})$

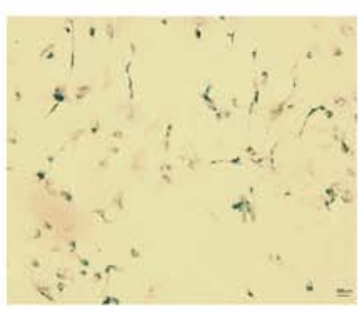

OA+PNS $(200 \mu \mathrm{g} / \mathrm{ml})$

E
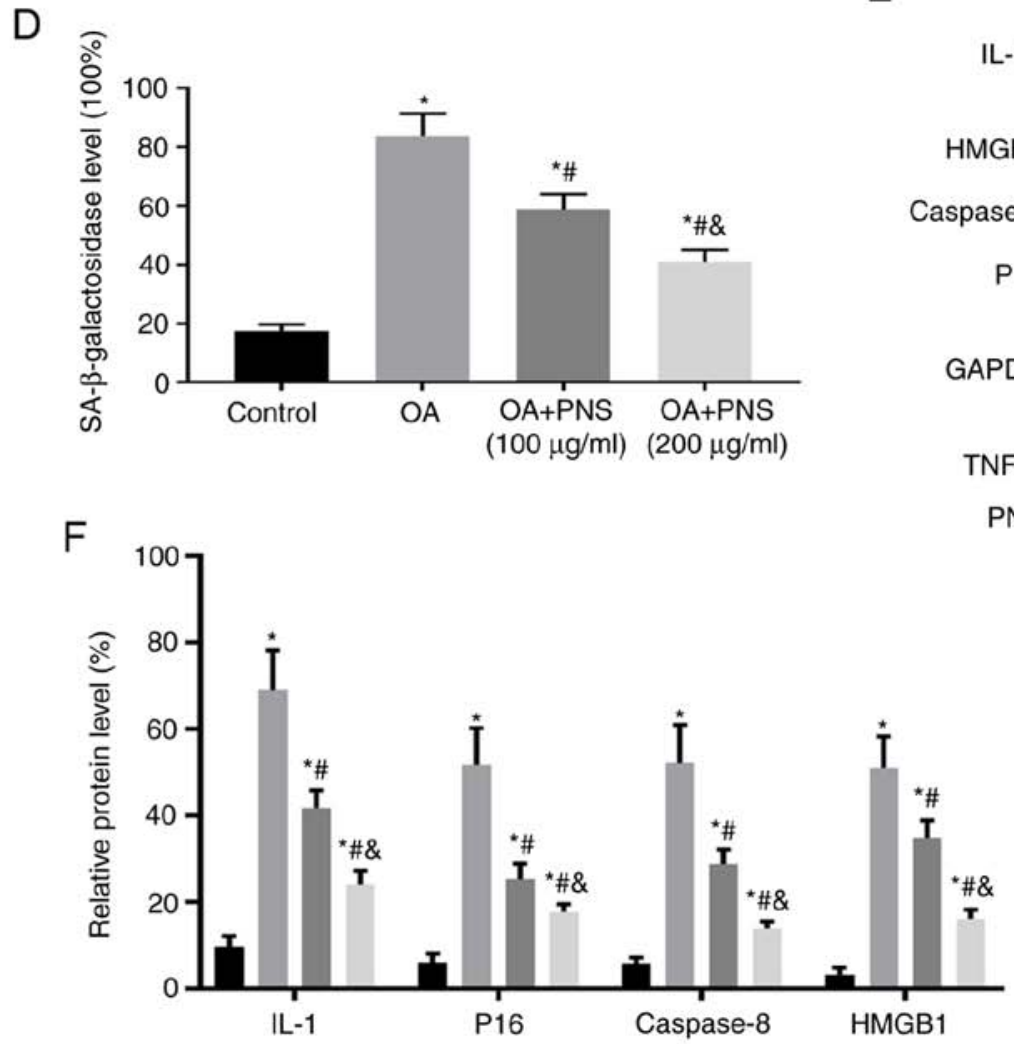

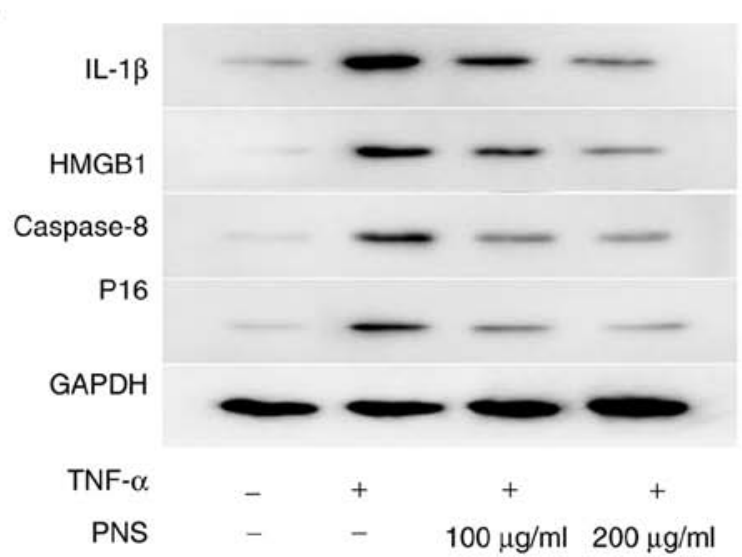

Figure 1. Chondrocytes isolated from rat cartilage were cultured (magnification, x40). (A) Identification of live chondrocytes by toluidine blue staining. Primary chondrocytes are stained blue-purple. (B) Cell viability at different concentrations of PNS. The data are expressed as the means \pm SD. ${ }^{*} \mathrm{P}<0.05$ vs. OA model chondrocytes. (C) Positive staining for vimentin (green), a marker of the senescence marker SA- $\beta$-galactosidase (magnification, x100) and (D) quantitative analysis of the numbers of positive cells. (E) Protein levels of IL-1, p16, caspase-8 and HMGB1 in chondrocytes were assessed by western blotting. Quantitative analysis of the protein levels of p16, IL-1, caspase-8 and HMGB1 based on the western blot results (F). The data are expressed as the means \pm SD. ${ }^{*} \mathrm{P}<0.05$ vs. normal chondrocytes; ${ }^{\#} \mathrm{P}<0.05$ vs. OA and $\mathrm{P}<0.05$ vs. OA+PNS $(100 \mu \mathrm{g} / \mathrm{ml})$ ( $\left.\mathrm{n}=3\right)$. OA, osteoarthritis; PNS, panax notoginseng saponins; SD, standard deviation; IL, interleukin; HMGB1, High mobility group protein B1.

effects of PNS on the metabolic activity of the ECM of cartilage were examined by immunohistochemistry, immunofluorescence and RT-PCR. As shown in Fig. 5, TNF- $\alpha$ treatment (OA) and PI3K-AKT-mTOR pathway agonist IGF-1 (OA+IGF-1+PNS) significantly reduced the mRNA level of the ECM gene, COL-II, and increased the mRNA level of the ECM degradation genes, MMP-3 and MMP-13 (Fig. 5D). PNS also inhibited the catabolic activity of ECM. Moreover, PNS antagonized the effect of the PI3K-Akt-mTOR pathway agonist IGF-1 to significantly inhibit COL-II degradation (Fig. 5A and D) and also decreased the expression levels of MMP-3 (Fig. 5B and E) 
A
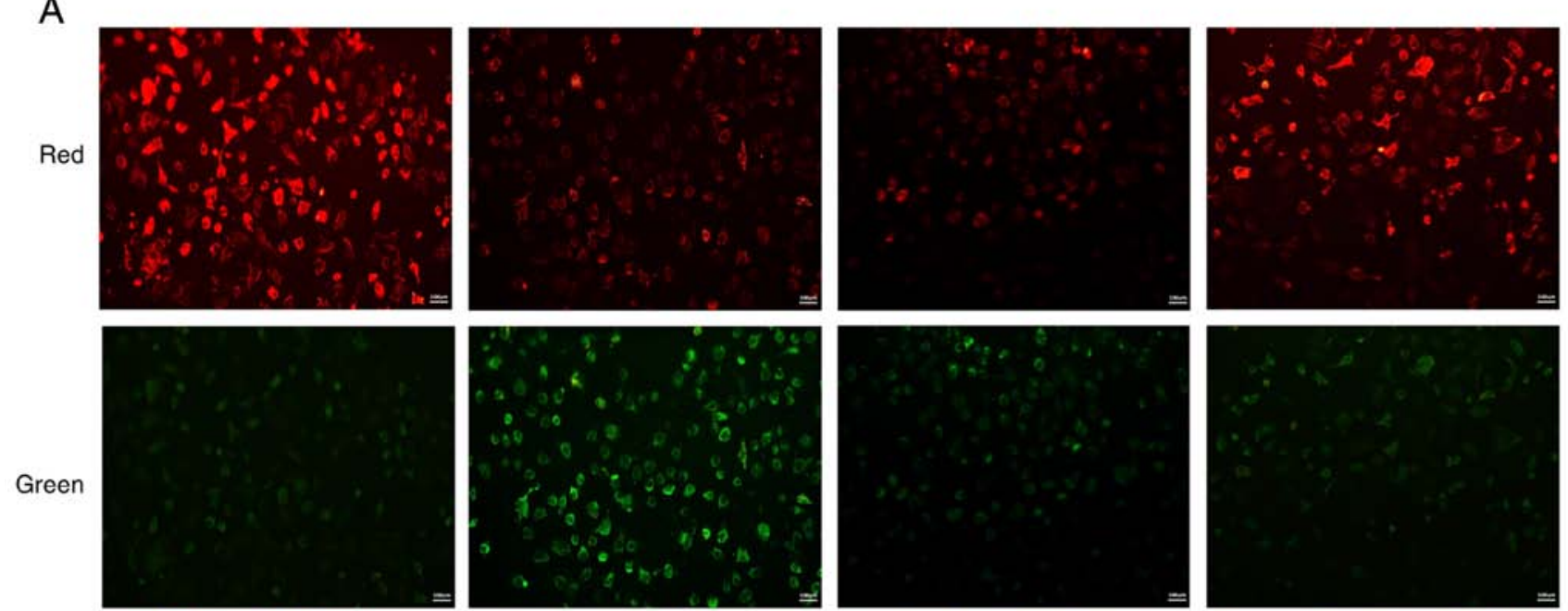

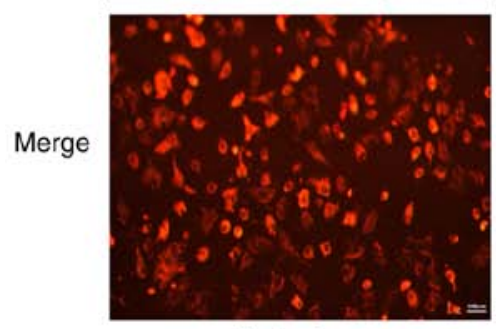

Control

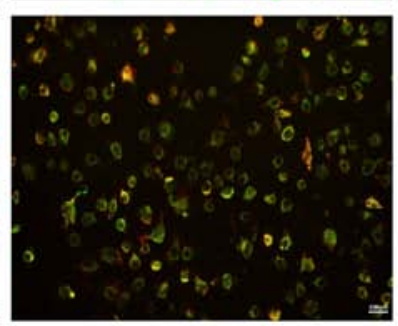

$\mathrm{OA}$

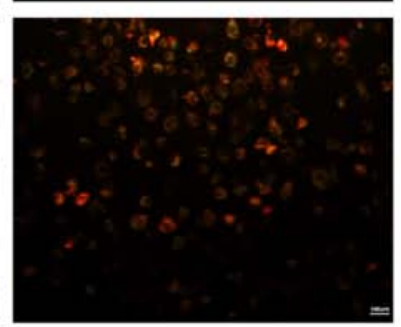

OA+PNS $(100 \mu \mathrm{g} / \mathrm{ml})$

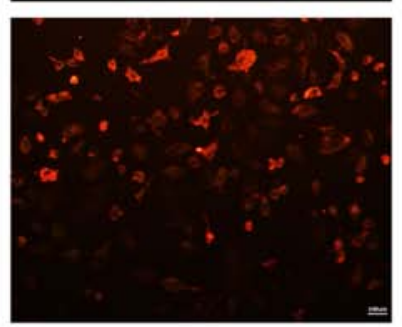

OA+PNS $(200 \mu \mathrm{g} / \mathrm{ml})$

B

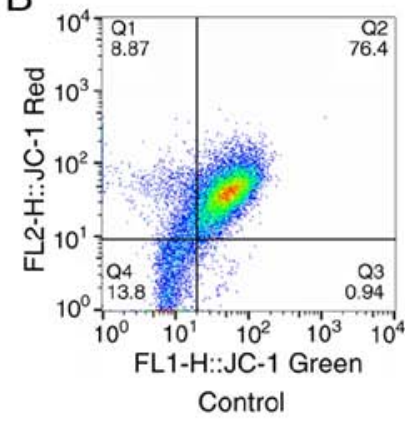

C
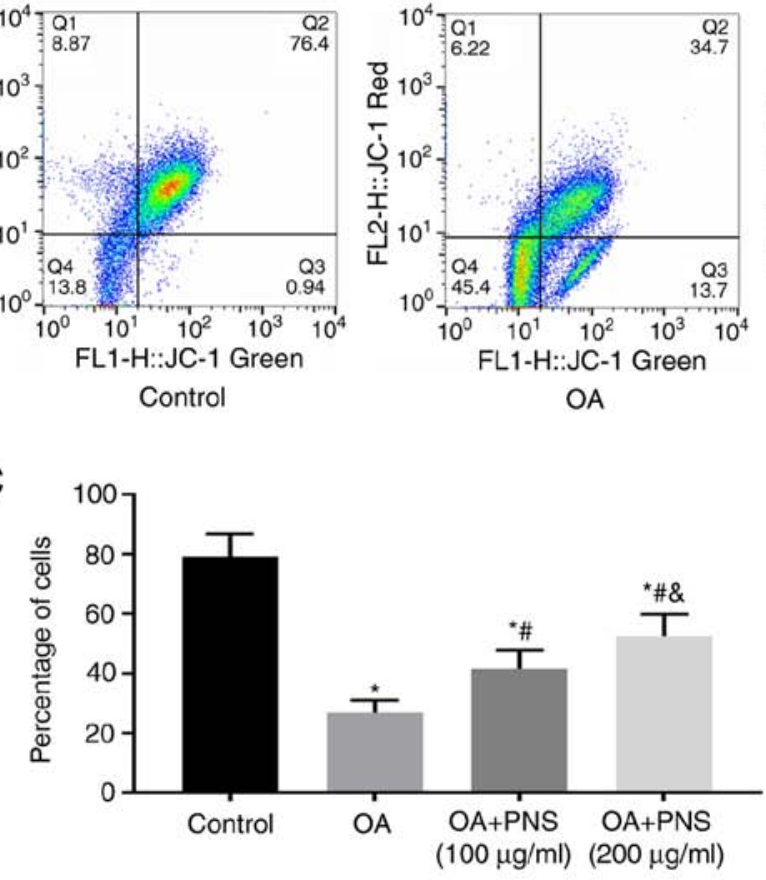

$\mathrm{OA}$

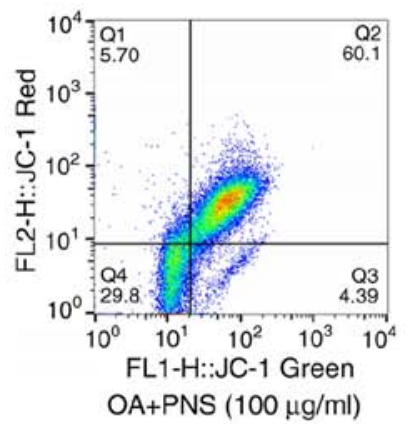

$\mathrm{D}$

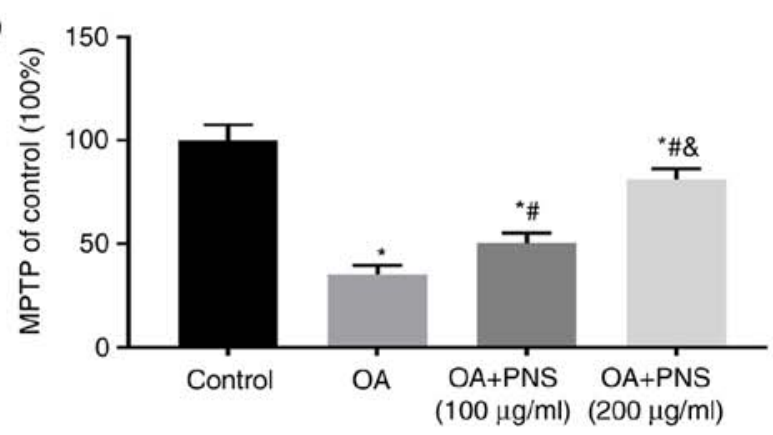

Figure 2. Detection of mitochondrial membrane-related indicators in chondrocytes. (A) Changes in MiMP of intracellular mitochondria in chondrocytes assessed by fluorescence microscopy (magnification, x200). Red fluorescence indicates normal MiMP and green fluorescence indicates the extent to which MiMP is reduced or lost. MiMP was examined by (B) flow cytometry and (C) quantified. (D) Flow cytometry was used to detect changes in MPTP. The data are expressed as the means \pm standard deviation. ${ }^{*} \mathrm{P}<0.05$ vs. normal chondrocytes; ${ }^{\#} \mathrm{P}<0.05$ vs. OA and ${ }^{\&} \mathrm{P}<0.05 \mathrm{vs}$. OA+PNS $(100 \mu \mathrm{g} / \mathrm{ml})(\mathrm{n}=3)$. MiMP, mitochondrial membrane permeability; OA, osteoarthritis; MPTP, mitochondrial membrane permeability conversion; PNS, Panax notoginseng saponins.

and MMP-13 (Fig. 5C and F); these results were consistent with the RT-PCR results (Fig. 5G).

PNS regulates the expression levels of PI3K/AKT/mTOR signaling pathway and ultrastructural changes in articular cartilage. The phosphorylation levels of PI3K, AKT and mTOR were significantly increased in the other groups compared to those in the control group. Compared with those in the OA group, IGF-1 increased the percentages of $\mathrm{p}-\mathrm{PI} 3 \mathrm{~K} / \mathrm{PI} 3 \mathrm{~K}$, p-AKT/AKT, p-mTOR/mTOR, whereas PNS significantly 
A

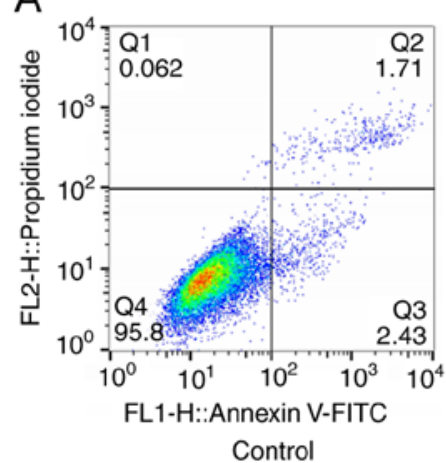

Control

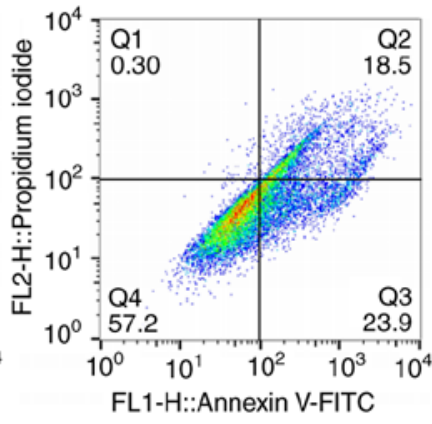

$\mathrm{OA}$

B
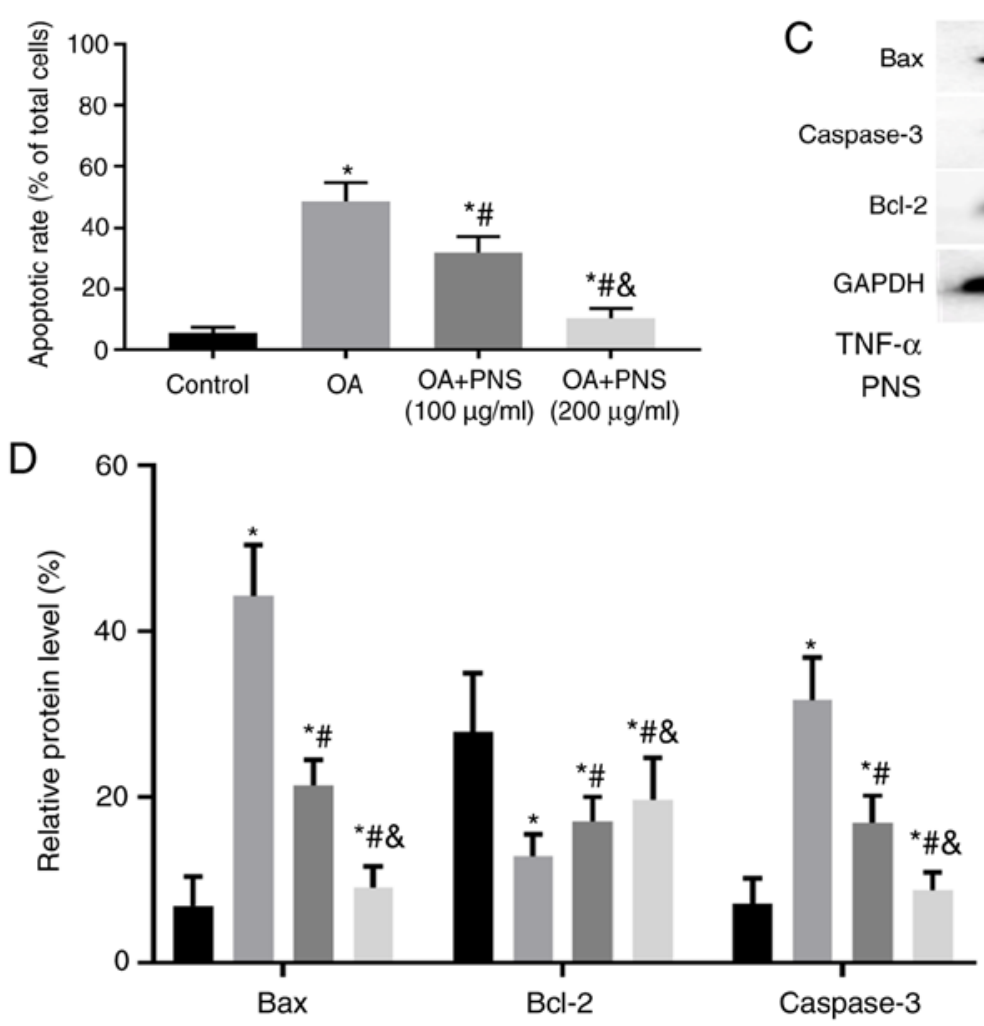

Figure 3. PNS reduces the level of apoptosis in OA chondrocytes. (A) Flow cytometry data showing changes in the apoptotic rate between the four groups and (B) quantification. (C) Western blot results show the protein expression levels of caspase-3, Bcl-2, Bax in chondrocytes and their (D) quantification. The data are expressed as the means \pm standard deviation. ${ }^{*} \mathrm{P}<0.05$ vs. normal chondrocytes; ${ }^{\#} \mathrm{P}<0.05$ vs. OA and ${ }^{\&} \mathrm{P}<0.05$ vs. OA+PNS (100 $\left.\mu \mathrm{g} / \mathrm{ml}\right)$ ( $\mathrm{n}=3$ ). FITC, fluorescein isothiocyanate; OA, osteoarthritis; PNS, Panax notoginseng saponins; TNF, tumor necrosis factor.

reduced them (Fig. 6A-D). Meanwhile, PNS can reduce the expression of apoptosis-related proteins and increase the expression of anti-degradation proteins (Fig. 6E and F). Ultrastructural changes in the chondrocytes were observed using transmission electron microscopy. The mitochondria showed a normal shape and the mitochondrial ridge was clearly visible in the control group; however, in the OA and $\mathrm{OA}+\mathrm{IGF}-1$ groups, the mitochondrial ridge disappeared and the mitochondria were swollen, indicating that the mitochondria were damaged and ruptured in these groups. These effects improved significantly in the PNS-treated experimental group (Fig. 6G). The present in vivo experiments showed that compared with those in the OA group, PNS significantly reduced the autophagy in chondrocytes and reduce apoptosis by regulating mTOR signaling via the PI3K-AKT pathway. This is consistent with the results of in vitro experiments.
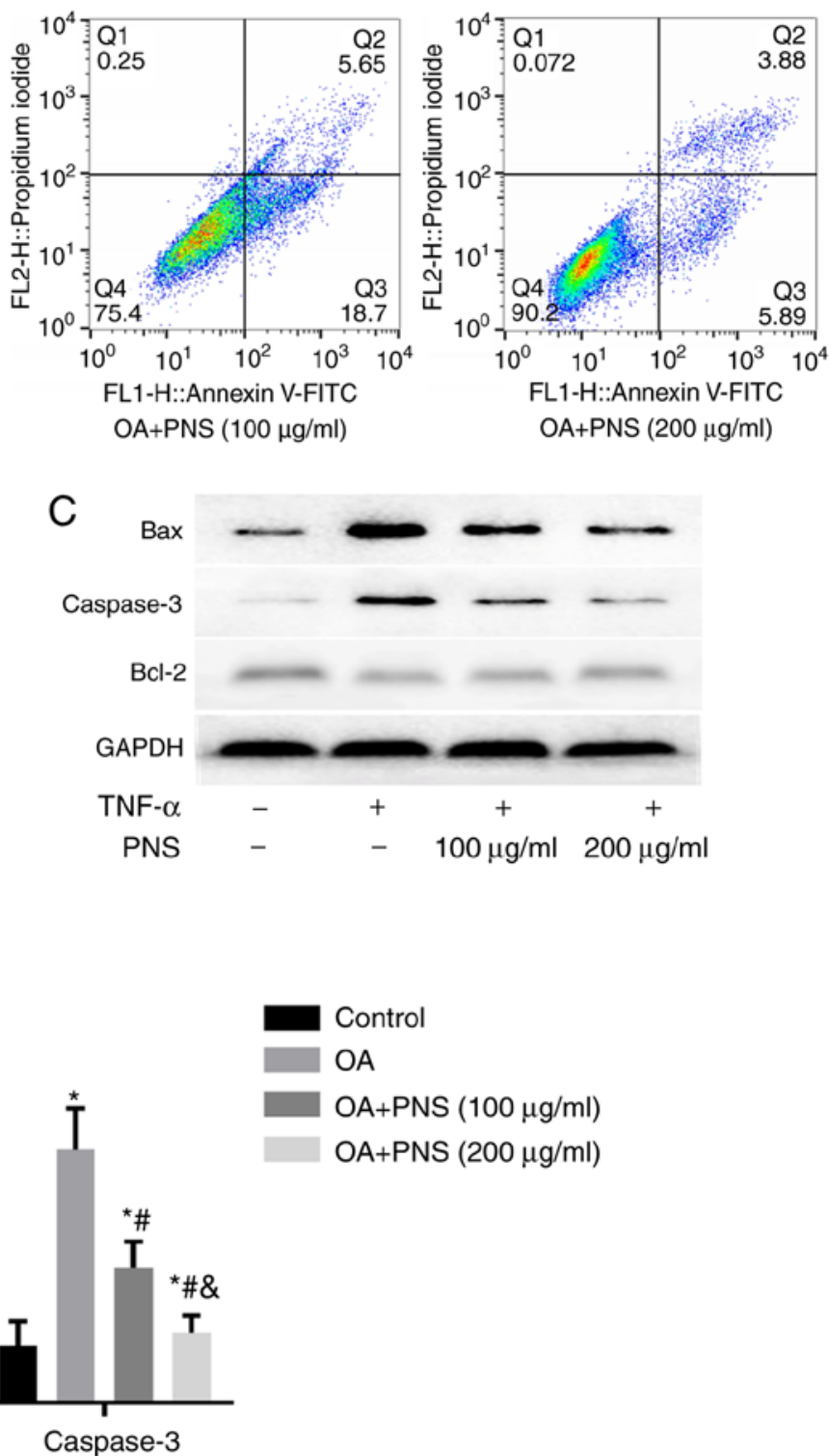

OA+PNS $(200 \mu \mathrm{g} / \mathrm{ml})$

\section{Discussion}

Compounds from some medicinal plants have various pharmacological effects and studies related to intra-articular injection of such compounds have become a research hotspot $(26,27)$. Previous studies have shown that PNS could inhibit cyclooxidase expression in macrophages and it has been speculated that PNS has anti-inflammatory and immunosuppressive effects in vitro $(28,29)$. Intraperitoneal injection of PNS significantly reduced the levels of TNF- $\alpha$ and IL- 6 in the serum of a liver fibrosis rat model (30). In another study, it was suggested that PNS had an anti-oxidant effect due to inhibition of reactive oxygen species formation and of the Bcl-2/Bax signaling pathway (31). Oxidative stress is an important factor that leads to cell damage and apoptosis. However, it is not clear 

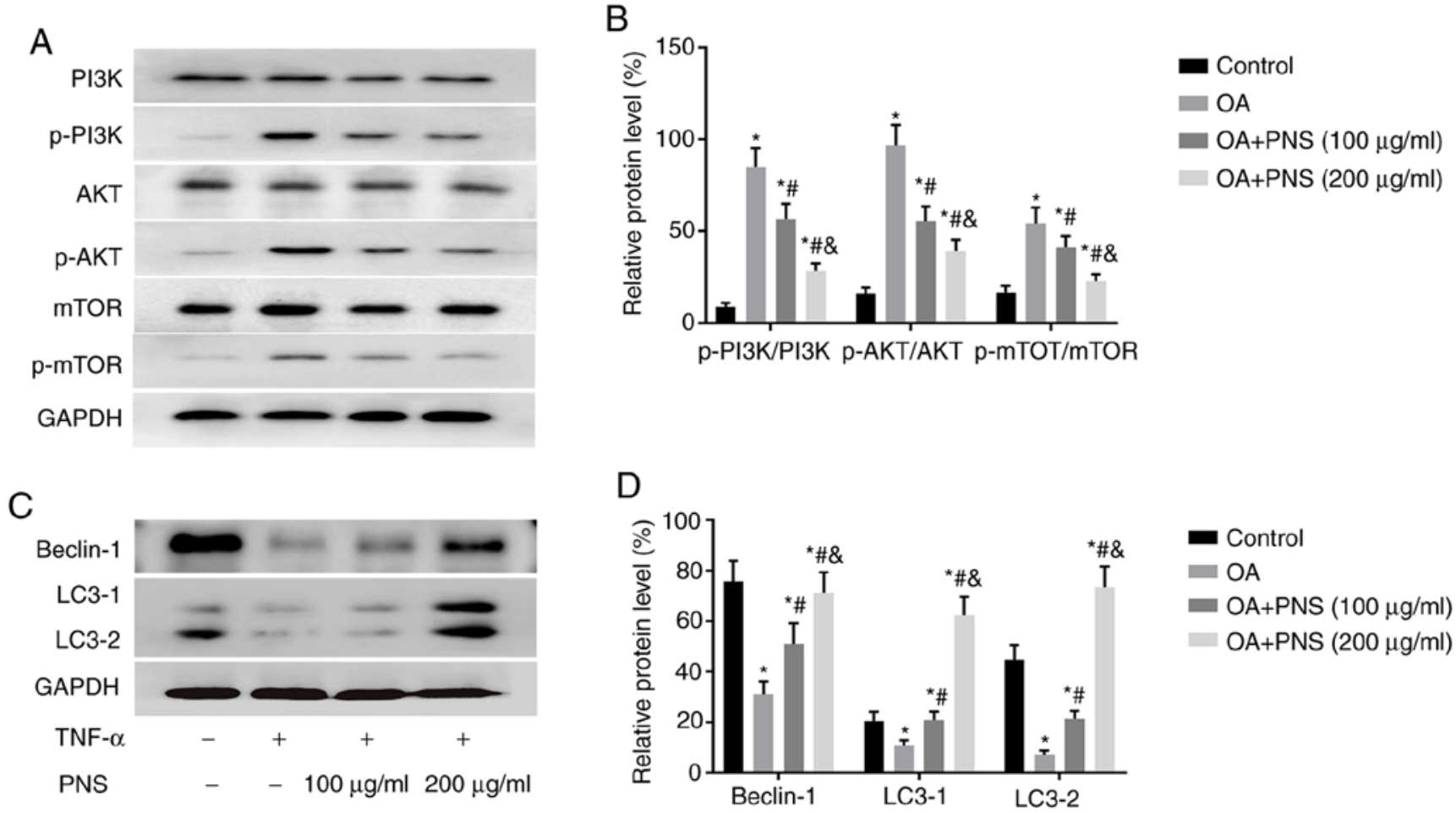

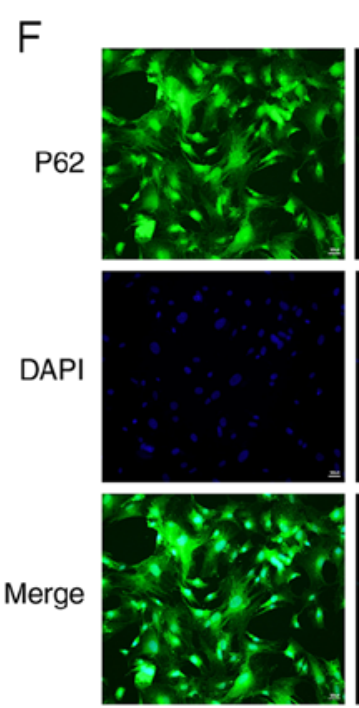

Control
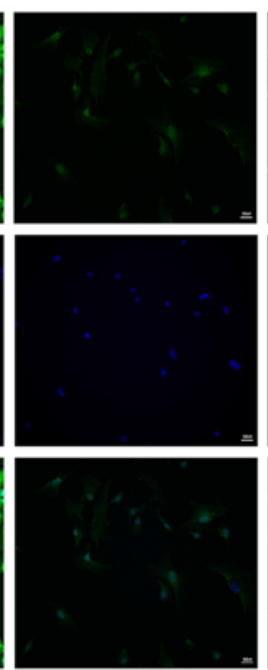

$O A$
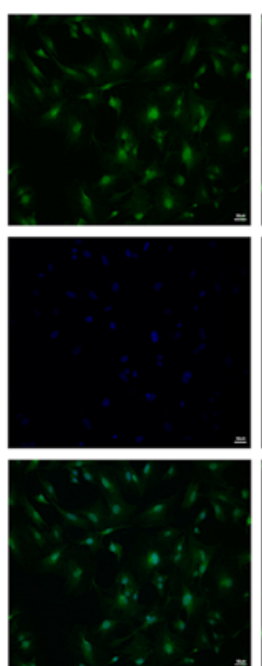

OA+PNS

$(100 \mu \mathrm{g} / \mathrm{ml})$
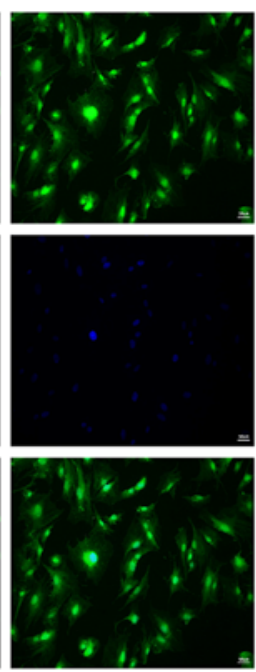

OA+PNS

$(200 \mu \mathrm{g} / \mathrm{ml})$

$E$

Figure 4. PNS regulates the PI3K/AKT/mTOR pathway to increase the expression levels of autophagy-related proteins. (A) Western blotting results showing protein levels of PI3K, p-PI3K, AKT, p-AKT, mTOR and p-mTOR in chondrocytes and were (B) quantified. (C) A western blot showing protein levels of LC3 and Beclin-1 in chondrocytes with (D) quantification. (E) Quantitative analysis of the LC3II/LC3I ratio based on western blot results. (F) Immunofluorescence staining showing the protein levels of P62 in chondrocytes along with $(\mathrm{G})$ quantification. The data are expressed as the means \pm standard deviation. ${ }^{*} \mathrm{P}<0.05$ vs. normal chondrocytes; ${ }^{\#} \mathrm{P}<0.05$ vs. OA and ${ }^{\circledR} \mathrm{P}<0.05$ vs. OA+PNS $(100 \mu \mathrm{g} / \mathrm{ml})(\mathrm{n}=3)$. PI3K, phosphatidyl inositol 3 kinase; p, phosphorylated; AKT, protein kinase B; mTOR, mammalian target of rapamycin; OA, osteoarthritis; PNS, Panax notoginseng saponins; TNF, tumor necrosis factor.

whether the above-mentioned anti-inflammatory effects can protect articular cartilage from destruction due to excessive secretion of pro-inflammatory factors during the progression of OA. In this study, PNS intervention reduced the secretion of cartilage SASP factors and reduced the extent of OA.

Autophagy and mitochondrial dysfunction are the main characteristics of cartilage degeneration caused by senes- cence (32). Due to cartilage degeneration and decreased autophagy, adaptability of cartilage cells to the external environment is significantly decreased, leading to cell damage, such as abnormal cellular metabolism, and ultimately to cell death. A study by Zhang et al (33) showed that increased autophagy signaling in the cartilage had a significant protective effect in an OA rat model that was constructed using resection of the medial meniscus of the right knee. This protective effect was 
A

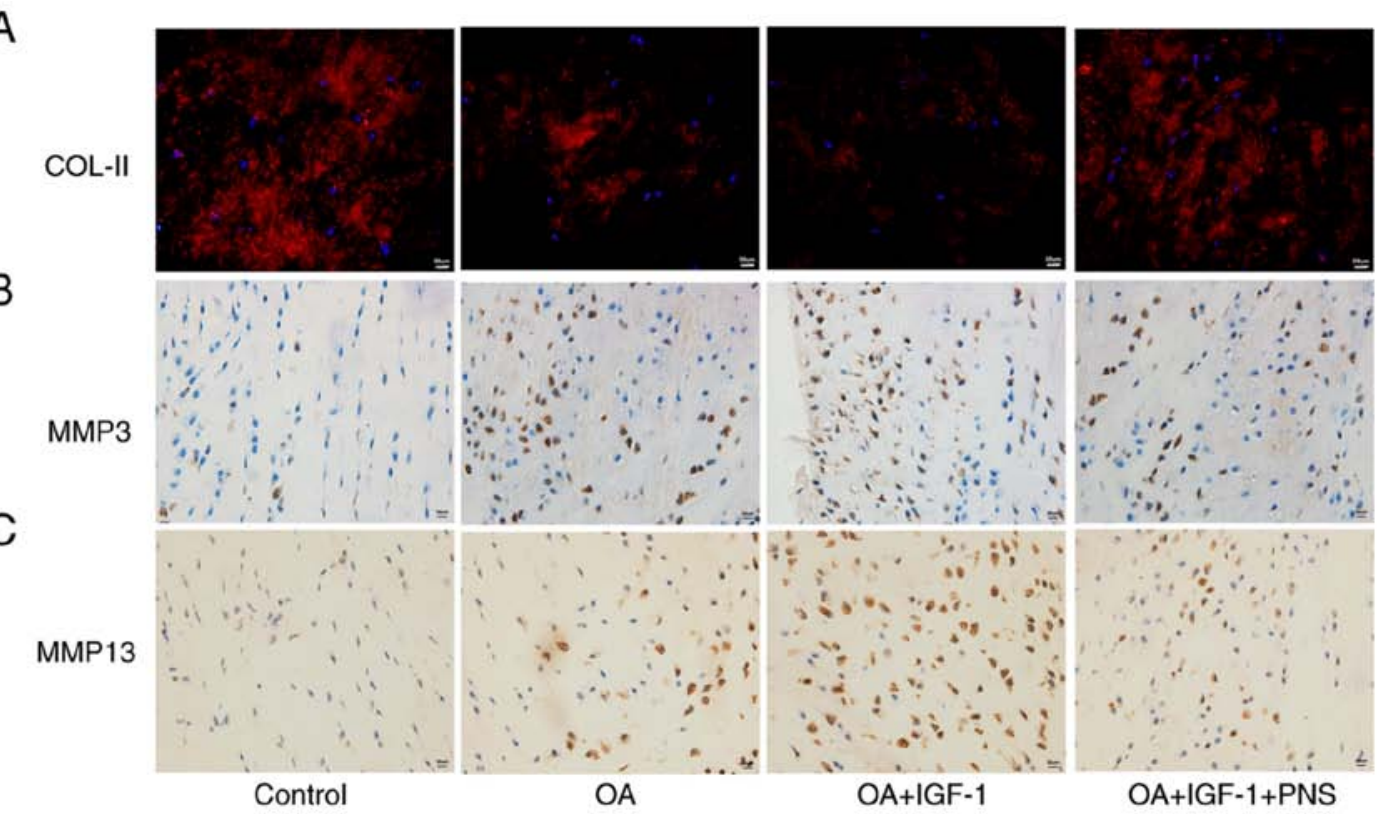

D

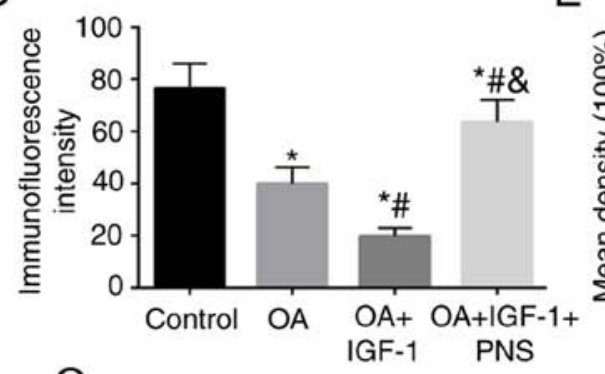

G

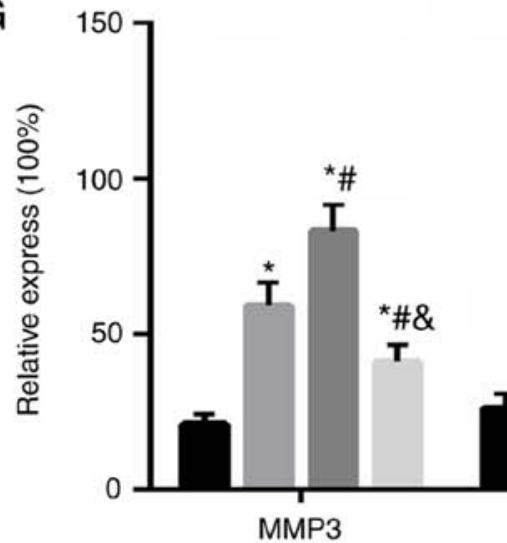

E

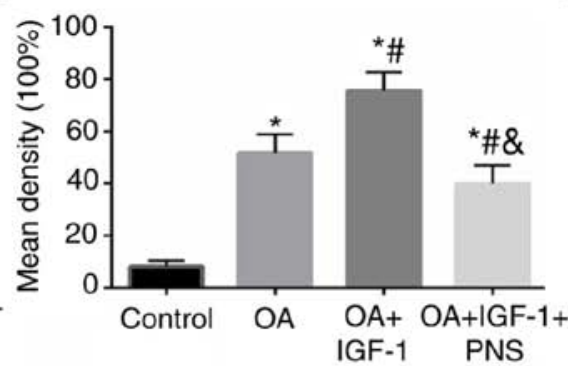

F

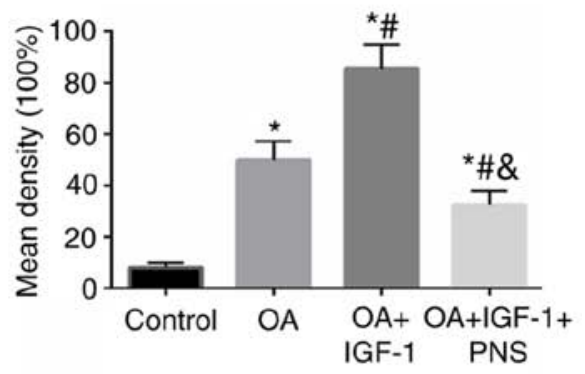

Figure 5. PNS regulates the expression levels of degeneration-related genes and ultrastructural changes in cartilage. (A) Immunofluorescence staining showing the protein levels of COL-II and the levels of (B) MMP-3 and (C) MMP-13 along with (D) quantification. Quantification of the (E) MMP-3 and (F) MMP-13 levels. Reverse transcription-PCR results are shown for COL-II, MMP-3 and MMP-13 (G). The data are expressed as the means \pm standard deviation ( $=3$ ). ${ }^{*} \mathrm{P}<0.05$ vs. control; ${ }^{\text {P }}<0.05$ vs. OA and ${ }^{\&} \mathrm{P}<0.05$ vs. OA+IGF-1. MMP, matrix metalloproteinase; OA, osteoarthritis; PNS, Panax notoginseng saponins.

related to degradation of the articular cartilage matrix and a decrease in apoptosis. In a previous study, the mitochondrial respiratory chain complex $\mathrm{V}$ inhibitor oligomycin was used to stimulate chondrocytes and the results showed dysfunctions in human chondrocyte mitochondria that led to reduced autophagy in chondrocytes. These observations suggest that inhibition of the mitochondrial respiratory chain inhibits autophagy (34). In the current study, it was demonstrated that TNF- $\alpha$ intervention promoted senescence in cartilage cells by inducing mitochondrial dysfunction and reducing autophagy, and that these effects could be reversed by the addition of PNS. These findings suggest that PNS may protect cartilage cells by regulating autophagy and delaying the progress of OA.

mTOR is a receptor of intracellular amino acids, ATP and hormones in human cells, and it is a major inhibitory regulator of intracellular autophagy $(35,36)$. In previous years, various studies have confirmed the important role of mTOR signaling in articular cartilage homeostasis and OA pathophysiology (37). Caramés et al (38) found that increased autophagy induced by the inhibition of mTOR signaling was 


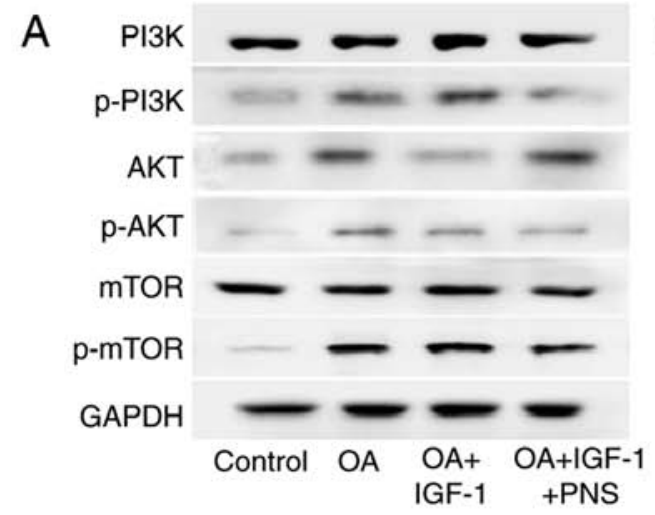

B

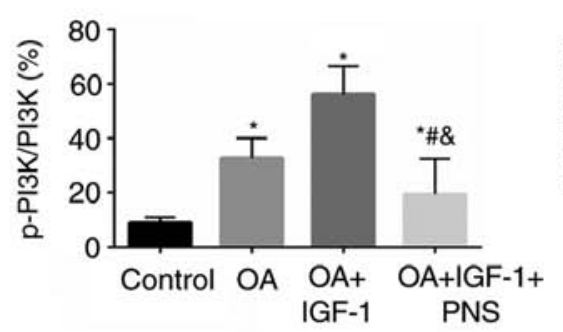

C

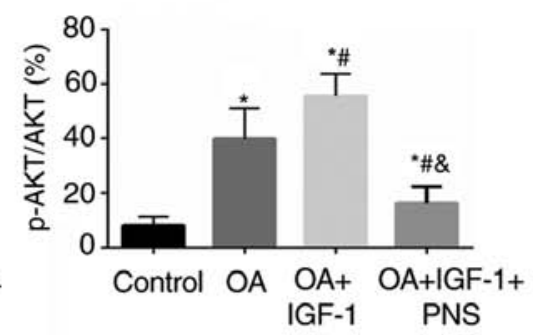

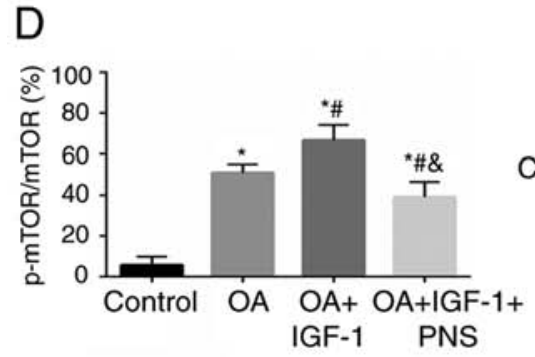

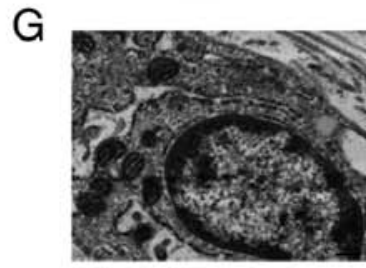

Control
E

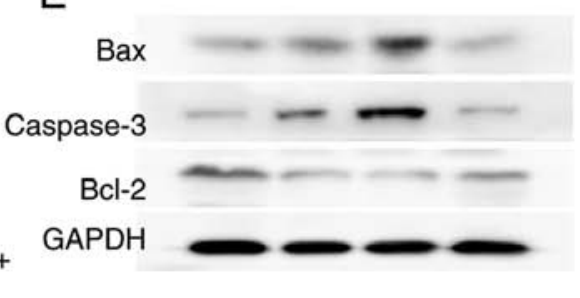

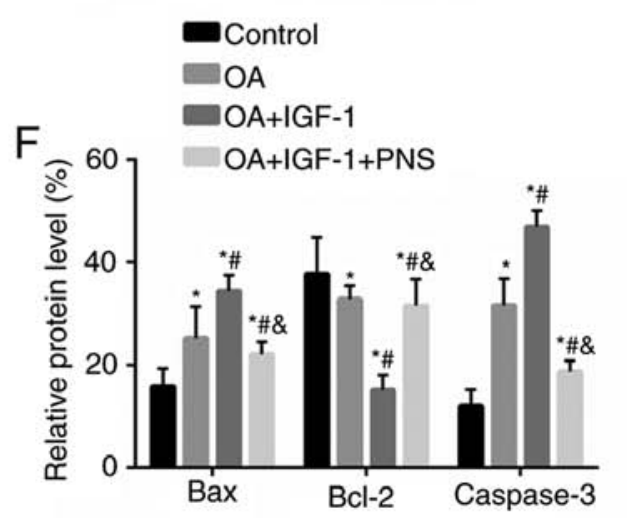

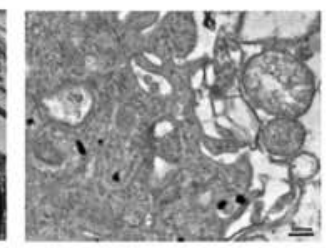

$\mathrm{OA}$

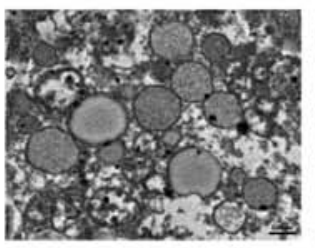

$\mathrm{OA}+\mathrm{IGF}-1$

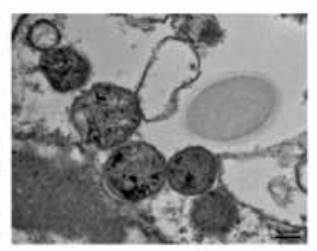

$\mathrm{OA}+\mathrm{IGF}-1+\mathrm{PNS}$

Figure 6. PNS regulates the expression levels of PI3K/AKT/mTOR signaling pathway and ultrastructural changes in articular cartilage. (A) Western blotting and quantification of (B) PI3K, p-PI3K, (C) AKT, p-AKT, (D) mTOR and p-mTOR levels were estimated using western blotting in rat articular cartilage and were quantified. (E) The expression of apoptosis-related proteins caspase-3 and Bax, anti-degradation proteins Bcl-2 and (F) quantification. (G) Transmission electron microscopy showing ultrastructural changes in chondrocytes (magnification, $\mathrm{x} 100,000)$. The data are expressed as the means \pm standard deviation $(\mathrm{n}=3)$. ${ }^{*} \mathrm{P}<0.05$ vs. control; " $\mathrm{P}<0.05$ vs. OA and $\mathrm{P}<0.05$ vs. OA+IGF-1. PI3K, phosphatidyl inositol 3 kinase; p, phosphorylated; Akt, protein kinase B; mTOR, mammalian target of rapamycin; OA, osteoarthritis; PNS, Panax notoginseng saponins.

associated with decreased ADAMTS-5 and IL-1 $\beta$ expression in articular cartilage. Rapamycin, a specific inhibitor of the mTOR signaling pathway, increased autophagy in cartilage cells in a mouse OA model and decreased the severity of OA (39). Rapamycin injection can lead to numerous adverse reactions, including diarrhea, weight loss, albuminuria, anemia, allergies, elevated serum cholesterol and triglycerides. In the current in vitro experiments (40), PNS demonstrated effects similar to those of rapamycin. The decrease in cartilage autophagy induced by TNF- $\alpha$ was reversed through the inhibitory effect of PNS on the PI3K/AKT/mTOR signaling pathway (41). In the present animal model, rats were treated with the PI3K/AKT/mTOR signaling pathway agonist, IGF-1; PNS was found have an antagonistic effect towards IGF-1. Therefore, the current study suggests that PNS can protect cartilage integrity by inhibiting PI3K/AKT/mTOR signaling pathway.

Previous studies showed that drugs that suppressed MiMP or pro-inflammatory factors often did not change the overall condition of OA $(42,43)$. However, regulating key factors affecting cell homeostasis, such as autophagy and preventing cell death and ECM destruction could limit OA progression $(44,45)$. Therefore, the regulation of autophagy via the mTOR signaling pathway might be a potential therapeutic target for OA. In this study, it was confirmed that PNS can reduce mitochondrial damage and senescence in cartilage cells by increasing autophagy via inhibition of the mTOR signaling pathway, ultimately delaying the development of OA in an animal model. Although the clinical implications are not yet clear, this study will provide a theoretical basis and a new concept for OA treatment.

\section{Acknowledgements}

The authors would like to thank Ms. Qiong Ding, Dr Pengchen Yu, Ms. Yingxia Jin and Ms. Lina Zhou (Central laboratory, Renmin Hospital of Wuhan University) for assistance with the flow cytometry analysis. 


\section{Funding}

This study was supported by the National Natural Science Foundation of China (grant no.81171760) and the Natural Science Foundation of Hubei Province (grant no. ZRMS2017000057).

\section{Availability of data and materials}

All data generated or analyzed during the current study are included in this published article.

\section{Authors' contributions}

YZ and HL designed the study. WC, GH, MC and SZ performed the experiments and analyzed the data. YZ, JL and $\mathrm{GH}$ contributed the essential reagents or tools and wrote the manuscript. All authors contributed to revising the manuscript and approved the final version to be submitted.

\section{Ethics approval and consent to participate}

The animal experiments and procedures were ethically approved by the Animal Care and Use Committee of Renmin Hospital of Wuhan University (Wuhan, China).

\section{Patient consent for publication}

Not applicable.

\section{Competing interests}

The authors declare that they have no competing interests.

\section{References}

1. Guilak F, Nims RJ, Dicks A, Wu CL and Meulenbelt I: Osteoarthritis as a disease of the cartilage pericellular matrix. Matrix Biol 71-72: 40-50, 2018.

2. Rahmati M, Mobasheri A and Mozafari M: Reply to 'Comment on: Inflammatory mediators in osteoarthritis: A critical review of the state-of-the art, prospects, and future challenges'. Bone 105: 311, 2017.

3. Englund M, Guermazi A, Gale D, Hunter DJ, Aliabadi P, Clancy $\mathrm{M}$ and Felson DT: Incidental meniscal findings on knee MRI in middle-aged and elderly persons. N Engl J Med 359: 1108-1115, 2008.

4. Prieto-Alhambra D, Judge A, Javaid MK, Cooper C, Diez-Perez A and Arden NK: Incidence and risk factors for clinically diagnosed knee, hip and hand osteoarthritis: Influences of age, gender and osteoarthritis affecting other joints. Ann Rheum Dis 73: 1659-1664, 2014.

5. Greene MA and Loeser RF: Aging-related inflammation in osteoarthritis. Osteoarthritis Cartilage 23: 1966-1971, 2015.

6. McCulloc K, Litherland GJ and Rai TS: Cellular senescence in osteoarthritis pathology. Aging Cell 16: 210-218, 2017.

7. Jeon OH, David N, Campisi J and Elisseeff JH: Senescent cells and osteoarthritis: A painful connection. J Clin Invest 128: 1229-1237, 2018.

8. Calcinotto A, Kohli J, Zagato E, Pellegrini L, Demaria M and Alimonti A: Cellular senescence: Aging, cancer, and injury. Physiol Rev 99: 1047-1078, 2019.

9. Wodarz D: Effect of stem cell turnover rates on protection against cancer and aging. J Theor Biol 245: 449-458, 2007.

10. Goessler UR, Bieback K, Bugert P, Heller T, Sadick H, Hörmann $\mathrm{K}$ and Riedel F: In vitro analysis of integrin expression during chondrogenic differentiation of mesenchymal stem cells and chondrocytes upon dedifferentiation in cell culture. Int J Mol Med 17: 301-307, 2006.
11. Hou A, Chen P, Tang H, Meng H, Cheng X, Wang Y, Zhang Y and Peng J: Cellular senescence in osteoarthritis and anti-aging strategies. Mech Ageing Dev 175: 83-87, 2018.

12. Ashkavand Z, Malekinejad H and Vishwanath BS: The pathophysiology of osteoarthritis. J Pharm Res 7: 132-138, 2013.

13. Muñoz-Espín D and Serrano M: Cellular senescence: From physiology to pathology. Nat Rev Mol Cell Biol 15: 482-496, 2014.

14. Levine B and Kroemer G: Autophagy in the pathogenesis of disease. Cell 132: 27-42, 2008.

15. LoPiccolo J, Blumenthal GM, Bernstein WB and Dennis PA: Targeting the PI3K/Akt/mTOR pathway: Effective combinations and clinical considerations. Drug Resist Update 11: 32-50, 2008.

16. Jones SA, Mills KH and Harris J: Autophagy and inflammatory diseases. Immunol Cell Biol 91: 250-258, 2013.

17. Chen Z, Jin T and Lu Y: AntimiR-30b inhibits TNF- $\alpha$ mediated apoptosis and attenuated cartilage degradation through enhancing autophagy. Cell Physiol Biochem 40: 883-894, 2016.

18. Choi AM, Ryter SW and Levine B: Autophagy in human health and disease. N Engl J Med 368: 651-662, 2013.

19. Green DR, Galluzzi L and Kroemer G: Mitochondria and the autophagy-inflammation-cell death axis in organismal aging. Science 333: 1109-1112, 2011.

20. Duan L, Xiong X, Hu J,Liu Y,Li J and Wang J: Panaxnotoginseng saponins for treating coronary artery disease: A functional and mechanistic overview. Front Pharmacol 8: 702, 2017.

21. HaipingZ,Ziping H, GuangwenL,SijiaZand YuminL:Therapeutic potential and cellular mechanisms of Panax notoginseng on prevention of aging and cell senescence-associated diseases. Aging Dis 8: 721-739, 2017.

22. Xu ZM, Li CB, Liu QL, Li P and Yang H: Ginsenoside Rg1 prevents doxorubicin-induced cardiotoxicity through the inhibition of autophagy and endoplasmic reticulum stress in mice. Int J Mol Sci 19: E3658, 2018.

23. Zhou P, Weijie X, He S, Sun Y, Meng X, Sun G and Sun X: Ginsenoside Rb1 as an anti-diabetic agent and its underlying mechanism analysis. Cells 8: 204, 2019.

24. Liu MW, Wei R, Su MX, Li H, Fang TW and Zhang W: Effects of Panax notoginseng saponins on severe acute pancreatitis through the regulation of mTOR/Akt and caspase- 3 signaling pathway by upregulating miR-181b expression in rats. BMC Complement Altern Med 18: 51, 2018.

25. Livak KJ and Schmittgen TD: Analysis of relative gene expression data using real-time quantitative PCR and the 2(-Delta Delta $\mathrm{C}(\mathrm{T})$ ) method. Methods 25: 402-408, 2001.

26. Kikuchi M, Matsuura K, Matsumoto Y, Inagaki T and Ueda R: Bibliographical investigation of complementary alternative medicines for osteoarthritis and rheumatoid arthritis. Geriatr Gerontol Int 9: 29-40, 2009.

27. Marcus DM: Therapy: Herbals and supplements for rheumatic diseases. Nat Rev Rheumatol 5: 299-300, 2009.

28. Wang T, Guo R, Zhou G, Zhou X, Kou Z, Sui F, Li C, Tang L and Wang Z: Traditional uses, botany, phytochemistry, pharmacology and toxicology of Panax notoginseng (Burk.) F. H. Chen: A review. J Ethnopharmacol 188: 234-258, 2016.

29. Rhule A, Navarro S, Smith JR and Shepherd DM: Panax notoginseng attenuates LPS-induced pro-inflammatory mediators in RAW264.7 cells. J Ethnopharmacol 106: 121-128, 2006.

30. Peng XD, Dai LL, Huang CQ, He CM, Yang B and Chen LJ: Relationship between anti-fibrotic effect of Panax notoginseng saponins and serum cytokines in rat hepatic fibrosis. Biochem Biophys Res Commun 388: 31-34, 2009.

31. Qiang H, Zhang C, Shi ZB, Yang HQ and Wang KZ: Protective effects and mechanism of Panax notoginseng saponins on oxidative stress-induced damage and apoptosis of rabbit bone marrow stromal cells. Chin J Integr Med 16: 525-530, 2010.

32. Youle RJ and Narendra DP: Mechanisms of mitophagy. Nat Rev Mol Cell Biol 12: 9-14, 2011.

33. Zhang Y, Vasheghani F, Li YH, Blati M, Simeone K, Fahmi H, Lussier B, Roughley P, Lagares D, Pelletier JP, et al: Cartilage-specific deletion of mTOR upregulates autophagy and protects mice from osteoarthritis. J Ann Rheum Dis 74: 1432-1440, 2015.

34. López de Figueroa P, Lotz MK, Blanco FJ and Caramés B: Autophagy activation protects from mitochondrial dysfunction in human chondrocytes. Arthritis Rheumatol 67: 966-976, 2015.

35. Jung CH, Ro SH, Cao J, Otto NM and Kim DH: mTOR regulation of autophagy. FEBS Lett 584: 1287-1295, 2010.

36. Xue JF, Shi ZM,Zou J and Li XL: Inhibition of PI3K/AKT/mTOR signaling pathway promotes autophagy of articular chondrocytes and attenuates inflammatory response in rats with osteoarthritis. Biomed Pharmacother 89: 1252-1261, 2017. 
37. Pal B, Endisha H, Zhang Y and Kapoor M: Mtor: A potential therapeutic target in osteoarthritis? Drugs R D 15: 27-36, 2015.

38. Caramés B, Hasegawa A, Taniguchi N, Miyaki S, Blanco FJ and Lotz M: Autophagy activation by rapamycin reduces severity of experimental osteoarthritis. Ann Rheum Dis 71: 575-581, 2012.

39. Kapoor M, Martelpelletier J, Lajeunesse D, Pelletier JP and Fahmi H: Role of proinflammatory cytokines in the pathophysiology of osteoarthritis. Nat Rev Rheumatol 7: 33-42, 2011.

40. Gianluigi Z, Paola T, Paolo R, Simona G, Luigino B and Antonio L: Systemic and nonrenal adverse effects occurring in renal transplant patients treated with mTOR inhibitors. Clin Dev Immunol 2013: 403280, 2013.

41. Fosang AJ and Little CB: Drug insight: Aggrecanases as therapeutic targets for osteoarthritis. Nat Clin Pract Rheumatol 4: 420-427, 2008.
42. Creel PA: Management of mTOR inhibitor side effects. J Clin J Oncol Nurs 13 (Suppl): S19-S23, 2009.

43. Mandl LA: Osteoarthritis year in review 2018: Clinical. Osteoarthr Cartilage 27: 359-364, 2019.

44. Miyaki S and Lotz MK: Extracellular vesicles in cartilage homeostasis and osteoarthritis. Curr Opin Rheumatol 30: $129-135,2018$.

45. Rai MF, Cotton R and Sandell LJ: Chemokines modulate chondrocyte homeostasis: Implications in osteoarthritis. Osteoarthr Cartilage 20 (Suppl): S240, 2012. 